\title{
Droplet traffic in microfluidic networks: A simple model for understanding and designing
}

\author{
Michael Schindler and Armand Ajdari \\ Laboratoire PCT, UMR “Gulliver" CNRS-ESPCI 7083, 10 rue Vauquelin, 75231 Paris cedex 05
}

\begin{abstract}
We propose a simple model to analyze the traffic of droplets in microfluidic "dual networks". Such functional networks which consist of two types of channels, namely those accessible or forbidden to droplets, often display a complex behavior characteristic of dynamical systems. By focusing on three recently proposed configurations, we offer an explanation for their remarkable behavior. Additionally, the model allows us to predict the behavior in different parameter regimes. A verification will clarify fundamental issues, such as the network symmetry, the role of the driving conditions, and of the occurrence of reversible behavior. The model lends itself to a fast numerical implementation, thus can help designing devices, identifying parameter windows where the behavior is sufficiently robust for a devices to be practically useful, and exploring new functionalities.
\end{abstract}

Injecting immiscible fluids into a microfluidic system has been found to permit the controlled generation of droplets or bubbles [1]. Rapidly, droplet microfluidics has developed beyond early observations because of its promises for a diversity of fields [2, 3]: studies of chemical kinetics and time-controlled synthesis [4], high-throughput screening [5], fabrication of remarkable colloidal objects [6], combinatorial chemistry [4], and even non-electronic coding or computing functions $[7,8]$. These tasks require mastering the generation of droplets, their traffic in potentially complex networks, and sometimes their splitting or merging. One can either employ passive means by relying on hydrodynamics and capillarity alone [1, 2, 4, 6, 7], or integrate active actuators (electric, dielectric, pneumatic) in the devices [2, 9]. Difficulties often arise even in simple geometries if the complex behavior of dynamical systems (period doubling, chaotic behavior, etc.) occurs [9, 10].

We here focus on the traffic of droplets only, without splitting or merging, in what we name dual networks, i. e. networks combining channels that can be accessed by droplets (transport channels) and others that cannot ("bypass" channels), see Fig. 1] This family of networks encompasses many recently proposed solutions [7, 8, 11, 12]. Building on recent works [11, 13], we introduce a simple numerical tool to analyze the collective motion of droplets in such a dual network. We show this tool to be powerful in both illuminating fundamentals ruling the traffic (including considerations of symmetry and reversibility) and as a means to predict the capability and robustness of a given design in performing a given function. In particular, we illustrate these ideas by analyzing three recently proposed devices as displayed in Fig. 1] a reversible encoding loop [8] (17), a ladder for synchronizing droplets between parallel channels [7] (1b), and a bypassed T-junction for symmetric splitting of a droplet train [12] (1).

Simple Model - We model both the flow of the continuous phase and the traffic of droplets in an arbitrary dual network of transport channels and bypasses, represented (a)

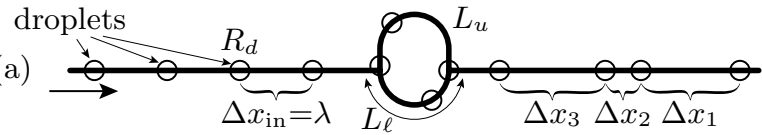

(b)

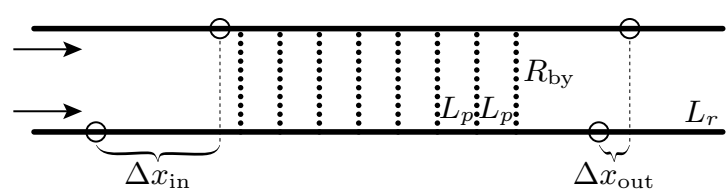

(c)

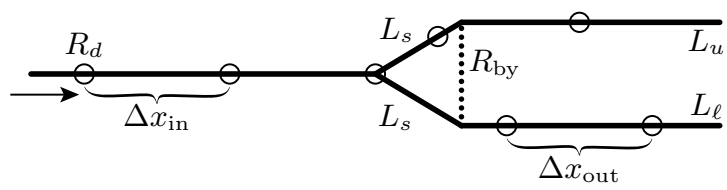

FIG. 1: Graph representations of three recently proposed dual networks: (a) a loop [7]; (b) a ladder [8]; (c) a bypassed Tjunction [12]. Full and dotted lines correspond to transport channels (accessible to droplets) and "bypass" channels (forbidden to droplets). Droplets are indicated by circles.

as a simple directed graph of nodes and links as in Fig. 1. The instantaneous flow state is given by a pressure $P_{\alpha}$ at each node and a total flowrate $Q_{i}$ in each channel. These variables are related by equations similar to Kirchhoff's rules for electrical circuits. Mass conservation at node $\alpha$, taking into account a possible external injection $Q_{\text {ext }}^{(\alpha)}$, reads

$$
\sum_{i \text { connected to } \alpha} \epsilon_{i} Q_{i}=Q_{\mathrm{ext}}^{(\alpha)}
$$

where $\epsilon_{i}= \pm 1$ account for the orientation of the link. In channel $i$ the flowrate $Q_{i}$ is related to the pressure difference between its two end nodes $\alpha_{\operatorname{in}(i)}$ and $\alpha_{\text {out }(i)}$,

$$
\Delta P_{i}=P_{\alpha_{\mathrm{in}(i)}}-P_{\alpha_{\mathrm{out}(i)}}=R_{i} Q_{i} .
$$

Equation (2) defines the hydrodynamic resistance $R_{i}$ of channel $i$. For flows without droplets at low Reynolds number it reduces to a constant value $\bar{R}_{i}$, proportional to the viscosity and to the channel length $L_{i}: \bar{R}_{i} \propto L_{i}$. 
Droplets or bubbles in the channel significantly modify the picture in a quite complex way [3, 11, 13]. A simplification occurs if the droplets are sufficiently distant from one another (typically a few channel diameters away) so that their flow perturbations do not interact. In the mentioned experiments the ratio of distance to diameter is approx. 10 (loop and ladder) and 5 (bypass junction). Each droplet then yields an additive increment $R_{d}$ to the resistance. In this paper, we make the further simplification that all droplets are equal and so are the cross-sections $S$ of the channels transporting droplets (these restrictions can be easily lifted in the numerical model), so that for $n_{i}$ droplets in channel $i$,

$$
R_{i}=\bar{R}_{i}+n_{i} R_{d},
$$

Identical droplets with no interaction move all at the same velocity $v_{i}$ in a channel with a given flow rate $Q_{i}$, which we write with a proportionality factor $\beta / S$,

$$
v_{i}=\beta Q_{i} / S \text {. }
$$

In general, both $R_{d}$ and $\beta$ will vary with $Q_{i}[13$, 14]. The simplifications in (3) and (44), however, have proved to hold in experimental realizations [11].

The flow state, consisting of all pressure and flowrate values, is uniquely determined by the set of linear equations (1) and (2) when complemented by appropriate boundary conditions at the end-nodes, i. e. specification of either the pressure or the externally injected flowrate. An important point is that this flow state persists until a droplet arrives at a node and enters another channel, thereby modifying the set of resistances. To describe droplet traffic, we thus track the locus of the droplets (modeled as points) along the channels. When a droplet arrives at a node where there are several channels to choose from, a selection rule is required. We focus here on locally symmetric T-junctions for which the droplets enter the channel with the instantaneous larger flow rate [1, 13].

Altogether, we propose the following algorithm: (i) Compute the pressures and flowrates for a given set of droplet positions using Eqs. (11) and (2); (ii) determine the droplet velocities according to Eq. (4); (iii) determine the next time when either a droplet arrives at a junction or a new one is injected, and advance all droplets to this time; (iv) decide which route the triggering droplet takes, update the channel resistances of the affected channels, and return to step (i).

Clearly, step (iii) is the big time saver that allows fast computations of rather involved histories. This scheme can be straightforwardly adapted to networks with channels of different cross-sections and to other junction geometries, but the present version is sufficient to revisit recently proposed devices and discuss fundamental issues.

Reversibility - Fuerstman et al. [8] presented a device that allows reversible "encoding" of information in interdroplet distances: Two long channels, used to store droplet series, are connected by a slightly asymmetric loop (Fig. 17). A regular train of droplets, injected through one channel into the loop, exits the loop as a complex modulated sequence. The interdroplet distances result from the "decisions" taken by the droplets when entering either branch of the loop. Each decision is affected by the presence of previous droplets in the loop. The slight asymmetry of the loop gives rise to periods of $2,3, \ldots 7$ in the outgoing sequence, depending on the initial interdroplet distance [8]. When we apply our simple model using parameters reasonably related to those of the experimental realization, we do obtain a comparable cascade of periods $(2,3,5,7,9$ etc.), as a characteristics of dynamical systems at work in this network [15].

A remarkable result by Fuerstman etal. [8] is that upon reversal of the flow direction, the starting sequence of droplets is restored, i. e. the system exhibits reversibility. This is far from being obvious, keeping in mind that the selection rule is not intrinsically reversible.

To investigate the question of reversibility further, in our numerics we send a periodic train of droplets through the loop until $N$ droplets have reached the other side, then invert the flow direction and send them back. The resulting $(N-1)$ interdroplet distances after this return trip through the loop are plotted in Fig. 2a for several values of the initial distance $\lambda$, ranging from 0 to the maximum for which two droplets interact, $\lambda_{\max }=$ $L_{\ell}\left[1+\left(\bar{R}_{\ell}+R_{d}\right) / \bar{R}_{u}\right]$ (see Fig. 1h for the parameters). The most striking feature of Fig. $2 \mathrm{a}$ is that it predicts windows of values of $\lambda$ for which the system behaves reversibly and other windows for which it does not (points not on the diagonal). It also sheds light on the impor-

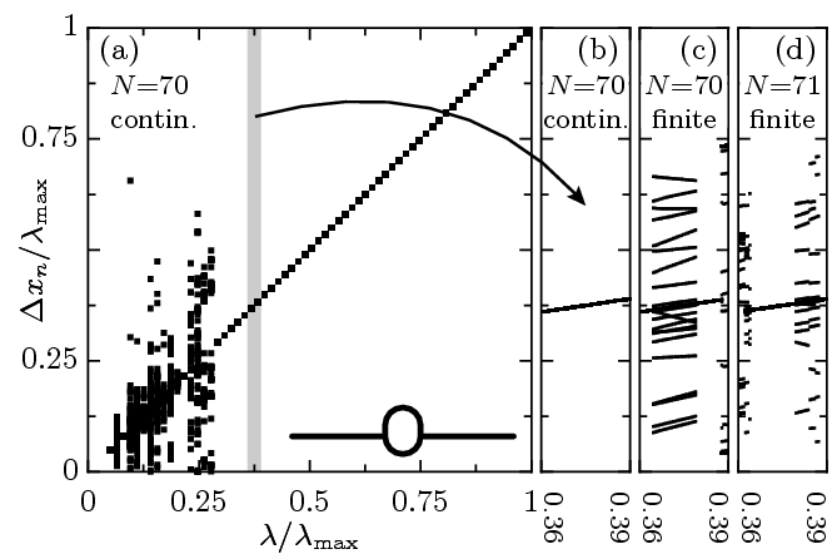

FIG. 2: Final distances $\Delta x_{n}$ between 70 droplets after a return journey through the loop of the device in Fig. 11, as a function of the initial period $\lambda$. Reversibility is observed where all 69 symbols collapse on the diagonal, see also the zoomed panel b. The results differ for a finite droplet train (panels c and d), where also the number of droplets matters. The parameters $L_{u} / L_{\ell}=1.112, R_{d} / \bar{R}_{\ell}=1.5$, are compatible with data from Ref. [8] [15]. 
tance of the precise conditions at flow inversion: For the same parameter interval [15], both full reversible behavior (Fig. 2b) and a complicated interrupted pattern (Fig. 2r) can be produced. In panels $2 \mathrm{a}$ and $2 \mathrm{~b}$ the droplets were perpetually injected, such that the loop was not empty when changing the flow direction. Conversely, in Fig. 2k and 2 d, a finite train of $N$ droplets was sent to and fro, with an empty loop in the meantime. This latter case is hardly reversible and depends on the precise number of droplets.

Let us underline that non-reversibility is deeply linked to the selection rule, and thus to the presence of junctions between transport channels. In a dual network with no such junctions, one expects the behavior to be reversible.

Reversibility and Symmetry of the Design - This observation leads us to a rather generic point regarding the kind of functions that a fore/aft symmetric device can achieve if the dynamics is reversible. A good support for this discussion is the recently proposed [7] symmetric ladder network of Fig. 1 b. Two transport channels are connected by a few (narrower) bypass channels. The device has been shown to perform the following function: Two droplets arriving with a given delay in the entrance channels leave the system with a much reduced distance, allowing synchronization between the two channels. Why is this remarkable? On one hand, (A) as the device is symmetric in design, if distances are reduced going one way, they should also be reduced going the reverse way. On the other hand, (B) the absence of junctions leading to "decision making steps" implies reversible dynamics, so that if the distance decreases one way, it should increase the other way: Such paradoxical statements are commonly used in low Reynolds number fluid dynamics to show that there can be no net effect. Here, experiments tell us otherwise.

Running our model immediately resolves the paradox in a manner that is practically relevant: The function can be achieved only if the boundary conditions are not sym-
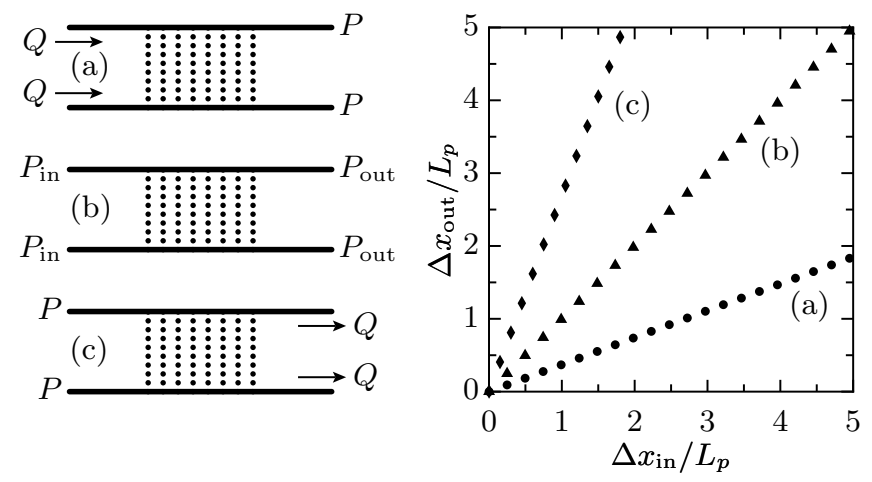

FIG. 3: Change of droplet misalignment in the ladder device. Only the boundary condition (a) leads to a contraction; fixed pressures on both ends (b) do not affect the offset, while it grows in (c). $R_{d} / \bar{R}_{p}=20, R_{\mathrm{by}} / \bar{R}_{p}=5, L_{r} / L_{p}=5$.

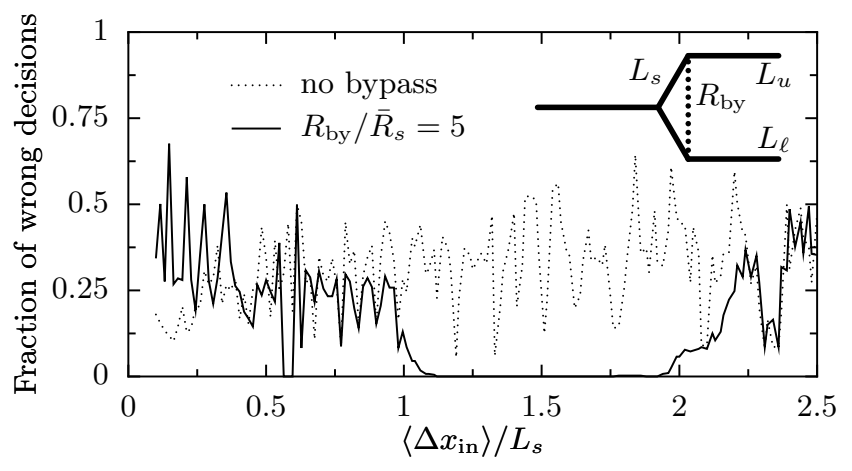

FIG. 4: Fraction of wrong (not strictly alternating) decisions in T-junctions with and without bypass (Fig. 1 1 ). The interval without errors around the average droplet distance $\left\langle\Delta x_{\text {in }}\right\rangle \approx 1.5 L_{s}$ corresponds to the experimental findings in Fig. 3 of Ref. [12]. The robustness of the behavior is challenged by an asymmetry $\left(L_{u} / L_{\ell}=1.01\right)$ and a random perturbation of the incoming distances (standard deviation is $5 \%$ of the average value). $R_{d} / \bar{R}_{s}=0.3$.

metric! For example, if we apply constant flow rates in the two entering arms and a fixed pressure at the two exiting nodes, we recover qualitatively the phenomenology described in Ref. [7] with distance reduction (see case a in Fig. 33). This reduction is not forbidden by the symmetry argument, as changing the sign of the flowrates and of the pressures yields a pattern of boundary conditions that is not the symmetric image of the initial situation, eliminating argument (A). In this new situation (case c in Fig. 3) the ladder acts as a distance expander rather than a distance contractor, in accordance with (B). Conversely, if we use fixed pressures at all end nodes, then the effect vanishes, as required by the symmetry argument $(\mathrm{A}+\mathrm{B})$ above (case b in Fig. 3). Our analysis thus clarifies the limits of use of this device as a synchronizing functional block in a network context.

At this stage, we have demonstrated that our model reproduces qualitatively and quantitatively the performance of existing devices. It illuminates the essential role of the imposed driving conditions [16], and it provides a priori indication as to the dynamic behavior (period doubling, reversibility, etc.) as a function of design parameters (geometrical symmetry) and operational parameters (droplets distance, etc.).

Designing Devices: Optimization and Robustness The speed of our numerical scheme permits fast optimization of identified designs. For example, we can scan the effect of different parameters of the ladder device (Fig. 3a), such as the number of bypasses, their resistance, their distance, or the resistance of the droplets. The parameter $R_{d} / \bar{R}_{p}$ has the strongest influence: the closer the bypasses, the better. Conversely, making the side arms of the transport channels $\left(L_{r}\right)$ very long, has a negative effect on the contraction. Increasing the number of bypasses from 3 to 10, or making them 100 times less 
resistant only weakly improves the contraction result.

Beyond this optimization in an ideal world, an essential point for suggested designs to turn into efficient working devices is their robustness to both fabrication errors and operational variations - virtues that are rarely quantified. We demonstrate the easy use of our tool to qualify the bypassed T-junction of Fig. 1k, proposed in Ref. [12]. The aim of this engineered junction is to provide reliable alternation of the channel chosen by incoming droplets. How it works is detailed in Ref. [12] - in short, the bypass channel imposes similar pressures at both its ends. A droplet having chosen one route is then able to "block" its channel such that the next droplet favors the other one due to its larger flowrate. With our numerical scheme we immediately recover the same parameter window for which the experimental design has been shown to be successful (cf. Fig. 3 in Ref. [12]).

We can systematically explore the robustness of the alternating behavior to imperfections of the device. These may consist of a "quenched" design asymmetry, such as a small systematic bias in the decision rule or a difference in arm lengths; or they may come as random fluctuations in the incoming droplet distance or in the pressures at the outlets. Figure 4 shows the effect of a random Gaussian perturbation of the incoming droplet distances, combined with a slight asymmetry of the channel lengths behind the bypass. Both perturbations lead to the complete failure of an unbypassed junction, whereas even a thin bypass robustly yields a perfect alternation in a significant window of parameters.

Playing with this analysis we find another remarkable property of the bypassed T-junction, namely noise reduction in the droplet distances. The distances in the two outgoing sequences have a smaller standard deviation than the incoming train (data not shown). This effect operates for a single junction and can be amplified by using a cascade of several junctions, connected in such a way that the average hydrodynamic resistances are balanced at each stage of the cascade [17]. At each level of the cascade the noise is reduced because the relative standard deviation of the distance between over-next droplets is only $1 / \sqrt{2}$ times that between adjacent droplets.

Conclusion - We have proposed a simple yet efficient fast numerical tool to analyze the traffic of droplets in "dual microfluidic" networks. Through an analysis of three recently proposed devices, we have shown the power of this tool for understanding fundamental issues, such as the role of the boundary conditions, of geometric symmetry and of the occurrence of reversible behavior. We have left aside physical effects, in particular capillarity, that could add further functionalities, such as trapping in tapered channels, splitting and merging of droplets at junctions, etc. However, we believe, that the presented method will stimulate further experiments to explore the validity of its central ideas. After such a validation step it will provide a guide for the design of devices, and for exploration of new functionalities. In particular, it lends its ideas to robust passive solutions that are likely steps for droplet (or bubble) microfluidics to practically come up to the hopes it has raised in many fields.

This work was supported by the French National Research Agency (ANR) via project Scan2, and we thank all members of this project for stimulating discussions.

[1] T. Thorsen, R. W. Roberts, F. H. Arnold, and S. R. Quake, Phys. Rev. Lett. 86, 4163 (2001).

[2] See the special issue of Lab Chip 4, 31N (2004); M. Joanicot and A. Ajdari, Science 309, 887 (2005); J. L. Steinbacher and D. T. McQuade, J. Polym. Sci. A: Polym. Chem. 44, 6505 (2006); H. Song, D. L. Chen, and R. F. Ismagilov, Angew. Chem. Int. Ed. 45, 7336 (2006).

[3] A. Günther and K. F. Jensen, Lab Chip 6, 1487 (2006).

[4] H. Song, J. D. Tice, and R. F. Ismagilov, Angew. Chem. Int. Ed. 42, 767 (2003).

[5] B. Zheng and R. F. Ismagilov, Angew. Chem. Int. Ed. 44, 2520 (2005).

[6] G. R. Yi, et al. Adv. Mat. 151300 (2003); D. Dendukuri, K. Tsoi, T. A. Hatton, and P. S. Doyle, Langmuir 21, 2113 (2005); T. Nisisako, T. Torii, T. Takahashi, and Y. Takizawa, Adv. Mat. 18, 1152 (2006).

[7] M. Prakash and N. Gershenfeld, Science 315, 832 (2007).

[8] M. J. Fuerstman, P. Garstecki, and G. M. Whitesides, Science 315, 828 (2007).

[9] H. Willaime, V. Barbier, L. Kloul, S. Maine, and P. Tabeling, Phys. Rev. Lett 96, 054501 (2006).

[10] P. Garstecki, M. J. Fuerstman, and G. M. Whitesides, Nature Physics 1, 168 (2005).

[11] W. Engl, M. Roche, A. Colin, P. Panizza, and A. Ajdari, Phys. Rev. Lett. 95, 208304 (2005).

[12] G. Cristobal, J.-P. Benoit, M. Joanicot, and A. Ajdari, Appl. Phys. Lett. 89, 034104 (2006).

[13] F. Jousse, R. Farr, D. R. Link, M. J. Fuerstman, and P. Garstecki, Phys. Rev. E 74, 036311 (2006); F. Jousse et al. Lab Chip 5, 646 (2005).

[14] F. P. Bretherton, J. Fluid Mech. 10, 166 (1961).

[15] We believe that the parameters in Fig. 2 $\mathrm{b}$ correspond to the experimental conditions used by Fuerstman et al. [8]. The cascade of forward periods in their Fig. 2B indicates an estimated value $L_{d} / \bar{L}_{\ell} \approx 1.5$, where we find a similar cascade. Their forward train had a period of 7 which we find in the interval $\lambda / \lambda_{\max } \in[0.3646,0.3837]$.

[16] In the special case of a single pair of end-nodes (e.g. the loop) the boundary conditions do not affect the sequence of events but only the pace at which they occur.

[17] International patent WO/2006/134274. 